\title{
Zur Kenntniss des Hämocyanins.
}

Von

Dr. M. Henze.

(Aus der physiologisch-chemischen Abtheilung der zoologischen Station zu Neapel.)

(Der Redaction zugegangen am 30. Juli 1901.)

Der Name Hämocyanin ist im Jahre 1878 durch die Arbeit Fredericq's ${ }^{1}$ ) "Recherches sur la physiologie du poulpe commun» in die Litteratur eingeführt worden, indem damit der im Blute von Octopus vulgaris vorkommende Eiweisskörper bezeichnet wurde. Gleichzeitig an das Hämoglobin der Wirbelthiere erinnernd, sollte mit diesem Namen auch die auffallende tiefblaue Farbe des sauerstoff haltigen, sogenannten Oxyhämocyanins angedeutet werden.

Das Hämocyanin war besonders interessant durch seinen Kupfergehalt; eine Thatsache, auf die bereits frühere Forscher aufmerksam gemacht hatten. Harless ${ }^{2}$ ) ist wohl als der Entdecker des Hämocyanins zu bezeichnen, der bereits im Jahre 1847 das Vorkommen von Kupfer im Blute von Octopus, ebenso von Helix pomatia, nachwies. Er zeigte auch, dass das Kupfer darin nicht etwa als Metallsalzlösung, sondern gebunden an ein Pigment existirte.

Wie nun im Blutfarbstoff der Wirbelthiere das Eisen eine so wichtige Function ausübt, scheint bei einer grossen Anzahl Kaltblütern das Kupfer diese Rolle zu übernehmen. Die Analogien mit dem Hämoglobin sind von Fredericq noch weiter ausgedehnt worden. Abgesehen davon, dass das Hämocyanin Sauerstoff $\mathrm{zu}$ binden und in Oxyhämocyanin überzu-

1) Arch. de zool. exp., VII, 1878, S. 535.

2) Müller's Arch., 1847, S. 148. 
gehen vermag, glaubte er auch, dass man mittelst Säuren eine Spaltung in einen neuen kupferfreien Eiweisskörper und einen krystallisirbaren, das gesammte Kupfer enthaltenden Körper bewirken könne. Der letztere würde also dem Hämatin, der erstere dem Globin entsprechen.

Die von Fredericq gemachten Beobachtungen sind später von verschiedenen anderen Forschern beschrieben worden. In der Hauptsache aber hat man sich begnügt, das Blut als Ganzes zu studiren. Man hat den Kupfergehalt von Neuem bestätigt und vor Allem eine grosse Anzahl Wirbelloser angegeben, die gleichfalls Kupfer in ihrem Blute führen. Wir finden $z$. B. bei Halliburton ${ }^{1}$ ) eine Tabelle dieser Thiere. In der gleichen Arbeit sind auch Einzelheiten über das Hämocyanin selbst angegeben. Es ist aber nicht ersichtlich, in wie weit mit reinen Hämocyanin gearbeitet wurde, da er das Blut von Crustaceen benutzte, welches nachweislich noch andere Eiweisskörper enthält. Denselben Einwand machte Fredericq ${ }^{2}$ ) einer Arbeit von Heim, ${ }^{3}$ ) der verschiedene der erwähnten Entdeckungen bestreitet, z. B. das Vorhandensein des Kupfers, das Sauerstoffbindungsvermögen, die Zersetzung durch Säuren etc.

Es konnte natürlich keine völlige Klarheit herrschen, so lange man nicht mit einem wirklich chemisch einheitlichen Körper arbeitete, und so musste zunächst auf diesen Punkt besonders geachtet werden. Fredericq gibt bereits in seiner citirten Arbeit an, dass das Octopusblut einzig und allein Hämocyanin enthalte, was er daraus schloss, dass der gesammte Eiweissgehalt bei einer bestimmten Temperatur zur Coagulation gebracht werden konnte und dass auch die fractionirte Alkoholfällung zu dem gleichen Resultat führte. Unter dieser Voraussetzung habe ich das Hämocyanin des Octopusblutes zu einem näheren Studium gewählt. Da dasselbe, wie unten gezeigt ist, auch zur Krystallisation zu bringen ist, so war ich im Stande, wirklich mit einem chemischen

1) J. of Physiology, VI, 300, 1885.

2) Comptes rendus des séances de l'académie des sciences. Paris. 115, 61. 1893 .

3) Ebenda 114, 771. 1892. 
Individuum $\mathrm{zu}$ arbeiten, so dass auch die analytischen Zahlen eine Berechtigung haben dürften.

Die Verblutung der Thiere geschieht am besten in der von Fredericq beschriebenen Weise, indem man den Pulp mit Nägeln auf einem Brett fixirt und nach Einführung der Canüle in die Arterie das Thier unter Wasser bringt. Nur auf diese Weise, die schon Fredericq selbst für etwas barbarisch erklärt, gelingt es, die grösstmöglichste Menge an Blut $\mathrm{zu}$ erhalten. Sowie das Thier schlecht athmet, gibt es weniger Blut, das missfarben aussieht. Die Farbe verwandelt sich jedoch sogleich in ein schönes tiefes Blau, sobald das Thier rhythmische Athembewegungen macht, ein entschiedenes Zeichen, dass die blaue Blutfarbe im Zusammenhang mit der Respiration und demnach mit der Sauerstoffaufnahme steht.

Die sonstigen Eigenschaften des Blutes an sich übergehe ich hier. Die Abhandlung Fredericq's gibt darüber genauen Aufschluss. Ich habe die gleichen dort citirten Beobachtungen gemacht, mein Augenmerk aber speciell auf das Hämocyanin gerichtet.

Krystallisation des Hämocyanins.

Das Hämocyanin war bisher nicht in krystallinischem Zustande bekannt. Gleichwohl darf man es mit zu den wenigen krystallisirbaren Eiweisskörpern rechnen, wenn man sich der Methode von Hofmeister resp. der zum Theil verbesserten von Hopkins und Pinkus bedient. Versetzt man das centrifugirte Blut mit soviel einer gesättigten Ammonsulfatlösung, dass noch keine bleibende Fällung eintritt, und überlässt es dann der Verdunstung in flachen Schalen, so scheidet sich nach Massgabe der Verdunstung das Hämocyanin krystallinisch aus.

Weit schöner und schneller gelingt die Krystallbildung bei Essigsäurezusatz. Das Blut wurde ebenfalls bis zur beginnenden Trübung mit Ammonsulfat versetzt, der Niederschlag eben wieder in Wasser gelöst und durch Zugabe einiger Tropfen verdünnter Essigsäure eine geringe Ausfällung von Neuem hervorgerufen. Schon nach halbtägigem Stehen hat sich der 
Niederschlag stark vermehrt und zu Boden gesetzt. Aus der decantirten Flüssigkeit lässt sich durch etwas Ammonsulfatlösung ein weiterer Krystallniederschlag erzielen. Der Krystallbrei bestand, wenn die Krystallisation gut gelungen war, aus mikroskopischen vortrefflich ausgebildeten Kryställchen. Auf krystallographische Bestimmung musste ich vor der Hand verzichten, doch zeigte sich schöne Doppelbrechung bei Anwendung polarisirten Lichtes. Bei der Methode der freiwilligen Verdunstung, wie sie ursprünglich Hofmeister anwandte, erschienen meistens $\mathrm{zu}$ Büscheln vereinigte Nädelchen. Nach der Vorschrift von Hopkins und Pinkus erhielt ich grössere einzelne Individuen von Prismenhabitus, die sich bei längerem Verweilen in der Mutterlauge vergrösserten. Auffälliger Weise verwandelten sich dieselben ein oder zweimal in eigenthümliche schollenartige Gebilde, von der Form eirunder Blättchen. Ihre Grösse war mitunter bedeutend und mit unbewaffnetem Auge wahrnehmbar. Ich glaube mich nicht $\mathrm{zu}$ täuschen, solche Blättchen von 3-4 mm. Durchmesser gesehen zu haben, nur liessen sie sich schwer aus dem Krystallbrei isoliren, indem sie zerbrachen. Zwischen Objectträger und Deckgläschen gebracht, wurden sie meist zerdrückt, man sah aber deutlich die zusammengehörigen Bruchstücke. Die Bedingung gerade für die Entstehung dieser Formen kann ich nicht angeben. Ich habe sie, wie gesagt, nur zweimal bei Präparaten, die längere Zeit gestanden hatten, beobachten können.

Die Krystalle lassen sich als solche schön fixiren, wie dies unter Anderen von Hofmeister, Gürber und Wichmann an anderen krystallisirbaren Eiweisspräparaten gezeigt worden ist. Man kann die Krystalle durch Erhitzen in der Mutterlauge oder durch Behandlung mit Alkohol coaguliren, ohne dass sie ihre Form einbüssen, nur dass man es höchst wahrscheinlich dann mit Pseudomorphosen zu thun hat. Ich habe eine grössere Anzahl Dauerpräparate dargestellt, um das Wachsthum der Krystalle von Tag zu Tag verfolgen zu können. Es geschah einfach in der Weise, in der man Bacterienpräparate anfertigt. Eine geringe Menge des Krystallbreies wurde auf ein Deckgläschen gebracht und 
über der Flamme angetrocknet. Nach dem Wegwaschen des Ammonsulfates wurde mit einer Farblösung, meist Fuchsin, tingirt und das Präparat in Canadabalsam eingebettet.

Obwohl es gelungen ist, krystallisirte Präparate von Neuem umzukrystallisiren, so war die zweite Krystallisation doch meist durch geringe amorphe Beimengungen verunreinigt.

Ferner scheint mir, dass die Krystallisation nur tadellos verläuft, wenn man frisches Blut und auch nur solches von gesunden Thieren verwendet.

Dialyse des krystallisirten Hämocyanins.

Zur weiteren Verarbeitung wurde der Krystallbrei auf einem Seidenfilter gesammelt und mit Ammonsulfatlösung (auf 7 Theile Wasser 3 Theile gesättigte Ammonsulfatlösung) nachgewaschen. Hierauf wurde derselbe in der eben ausreichenden Menge Wasser gelöst und zunächst gegen fliessendes, dann gegen destillirtes Wasser dialysirt, bis auch nicht die geringste Schwefelsäurereaction mehr eintrat. Diese mit Luft geschüttelte blaue Lösung enthält das Hämocyanin in reinem Zustande. Die Reaction ist völlig neutral. Es wurde einmal die Concentration einer solchen Lösung bestimmt: $25 \mathrm{ccm}$. wurden mit Alkohol coagulirt, gewaschen, erst im Vacuum und dann bei $110^{\circ}$ getrocknet. Das Gewicht des Coagulums betrug $0,8395 \mathrm{~g}$, d. h. die Lösung war 3,36 \% $/ 0$ g. Ehe ich auf die sonstigen Eigenschaften des Hämocyanins eingehe, muss noch auf ein Verhalten aufmerksam gemacht werden. Mehrere Male hatte sich in den Dialysirschläuchen ein sehr geringer Antheil des Hämocyanins ausgeschieden. Nun führt Halliburton an, dass bei der Dialyse das Hämocyanin ausgeschieden werde und rechnet es deshalb zu den Globulinen. Ich habe aber bei der Dialyse des Octopusblutes selbst, und ich habe diesen Versuch gewiss an die zehn Mal wiederholt, nie eine Ausscheidung beobachten können. Auch Fredericq gibt nichts dergleichen in seiner Arbeit an, trotzdem er auch dialysirt hat. Jedenfalls sollte auch beim Verdünnen einer Hämocyaninlösung mit viel Wasser eine Ausfällung eintreten, wenn ein echtes Globulin vorläge. Dies ist aber nicht der Fall; weder Blut 
selbst, noch eine reine Lösung krystallisirten Hämocyanins verhalten sich so. Es ist übrigens eine bekannte Erscheinung, dass reine Eiweisslösungen bei der Dialyse mitunter kleine Mengen ausscheiden.

Der geringe, durch Dialyse ausgefällte Antheil des Hämocyanins ist übrigens fast völlig löslich, sowohl in Neutralsalzen, als auch in einer Spur Säure. Ich habe deshalb versucht, ihn zur Krystallisation zu bringen, indem ich ihn in Ammonsulfat löste und mit gesättigter Ammonsulfatlösung versetzt zur Krystallisation in eine Schale gab. Es schieden sich in der That schöne Krystalle, völlig mit den Formen des Hämocyanins übereinstimmend, $a b$.

Bestimmung der Coagulationstemperatur.

Die Temperaturen für den Coagulationspunkt werden ziemlich verschieden angegeben. Fredericq sah nach seiner ersten Arbeit das Blut bei $65^{\circ}$ opalescent werden. Die eigentliche Coagulationstemperatur verlegt er auf $74^{\circ}$. In einer zweiten Mittheilung ${ }^{1}$ ) ist $69^{\circ}$ angegeben. Das Blut ist allerdings mit $10 \%$ Chlornatrium versetzt. Krukenberg's und Halliburton's Angaben liegen in ähnlichen Grenzen.

Ich habe drei verschiedene Bestimmungen ausgeführt. $\mathrm{Zu}$ den beiden ersten wurden reine dialysirte 1,5\% \% ige Lösungen von krystallisirtem Hämocyanin benutzt. Die dritte gleich vorbereitete Lösung erhielt ausserdem einen Zusatz von Kochsalz, sodass der Gehalt derselben daran 5\% betrug. Die Bestimmungen wurden in einem Beckmann'schen Gefrierpunktsapparat ausgeführt. An Stelle des äusseren, sonst mit Eis beschickten Gefässes diente ein Becherglas, das vorsichtig erhitzt werden konnte.

Die Coagulation tritt sehr langsam ein. Von $68-70^{\circ}$ beobachtete ich langsam stärker werdende Opalescenz. Deutliche Flockung und schliessliche vollkommene Fällung erfolgte bei $71-72^{\circ}$. Die Lösung bleibt unverän dert neutral. Das Filtrat ist eiweissfrei. Der Chlornatriumzusatz bei dem dritten

1) Comptes rendus des séances de l'académie des sciences. Paris. LXXXVII, 996. 
Versuch bedingte keine wahrnehmbare Verschiebung des Coagulationspunktes.

\section{Reactionen des Hämocyanins.}

a) Aussalzen: Die gewöhnlichen, zum Aussalzen der Eiweisskörper verwendeten Neutralsalze fällen das Hämocyanin nur unvollständig. Eine Ausnahme bildet allein das Ammonsulfat. Doch bewirkt auch dieses erst nach Sättigung in Substanz und längerem Stehen damit Totalfällung. Die unter€ Grenze für Ammonsulfat liegt bei $3, \tilde{5}$.

Halliburton führt an, nach Schütteln des Blutes bis zu 36 Stunden mit gepulvertem Magnesiumsulfat eine vollständige Fällung erhalten $\mathrm{zu}$ haben. Es ist wohl zweifellos, dass dabei mechanische Ursachen mitgewirkt haben, jedenfalls kann man jetzt nicht mehr aufrecht erhalten, das Hämocyanin durch Magnesiumsulfat ausgesalzen wird, und dies mit als Grund für seine Globulineigenschaften anführen. Ich habe durch tagelanges Stehen der Hämocyaninlösung mit Magnesiumsulfat in Substanz und öfterem Bewegen des Gefässes immer nur unvollkommene Fällung erzielen können. Fredericq erwähnt, dass Magnesiumsulfat überhaupt keine Fällung erzeuge, und auch andere Gründe veranlassen ihn, entgegen Halliburton, das Hämocyanin nicht zu den Globulinen zu zählen. Als Globulin sollte das Hämocyanin auch durch Ammonsulfat bei höchstens 4,6 gefällt werden, was nicht der Fall ist.

b) Eiweissreactionen: Die typischen Eiweissreactionen liefert das Hämocyanin sämmtlich. Es entstehen also z. B. Fällungen mit Phosphorwolframsäure, Phosphormolybdänsäure, Ferrocyankalium beim Ansäuern, Pikrinsäure, Tannin etc. Die Xanthoprotein- und Millon'sche Reaction ist deutlich. Sehr hübsch ist die Biuretreaction. Man kann dieselbe ohne den Kupfersalzzusatz ausführen, denn das Hämocyanin enthält eben schon Kupfer. Man hat nur nöthig, etwas stärkeres Alkali zu einer Lösung von Hämocyanin zu geben, um fast augenblicklich eine schöne rothviolette Färbung zu erhalten. Es ist dies zugleich ein sehr einfacher Nachweis für den Kupfergehalt des genannten Eiweisskörpers. 
Die Schwefelbleireaction ist sehr deutlich.

c) Schwermetallsalze bewirken in reinen Hämocyaninlösungen sofortige bleibende Fällungen. Eine Ausnahme macht entgegen Fredericq's Angabe das Bleiacetat. Es fällt zwar bei vorsichtigem Zusatz, der Niederschlag löst sich aber im Ueberschuss wieder klar auf. Vielleicht lässt sich diese Eigenschaft zur Trennung des Hämocyanins von anderen Eiweisskörpern verwenden.

d) Verhalten zu Säuren und Alkalien: Schon Fredericq beobachtete, dass stärkere Mineralsäuren flockige Fällung erzeugen. Dasselbe soll nach Halliburton starke Essigsäure bewirken. Ich habe selbst durch Eisessig keine Ausfällung erhalten. Es tritt nur Syntoninbildung ein.

Anders verhält sich das reine Hämocyanin, wenn man mit dem Zusatz der Säure sehr vorsichtig verfährt und dieselbe ganz verdünnt anwendet. Das Hämocyanin wird dann unverändert, aber nur zum Theil ausgefällt. Ein weiterer geringer Zusatz von Säure löst wieder völlig klar. Salzsäure, Salpetersäure, Schwefelsäure und Essigsäure verhalten sich darin völlig gleich. Die verdünnten Lösungen von Soda, Aetzalkali oder Ammoniak erzeugen selbst bei grösster Vorsicht keine Fällungen.

Dass Hämocyanin ebenfalls nur unvollkommen durch längeres Einleiten von Kohlensäure gefällt wird, erwähnt Krukenberg. Ich habe dasselbe beobachtet.

Elementare Zusammensetzung des Hämocyanins.

$\mathrm{Zu}$ den Analysen dienten drei mit besonderer Sorgfalt hergestellte Präparate. Die Kontrollanalysen wurden nie mit dem gleichen Präparat ausgeführt. Nur Krystalle ohne jegliche amorphe Beimengung kamen in Anwendung, die nach der Auflösung gegen destillirtes Wasser dialysirt wurden. Die erhaltene salzfreie Lösung wurde entweder durch Alkohol oder bei eben schwach essigsaurer Reaction durch Hitze coagulirt. Der Niederschlag wurde mit Wasser, Alkohol und Aether gewaschen und zunächst im Vacuum getrocknet. Er nimmt eine tief blaugrüne Farbe an. Nachdem er fein zerrieben worden war, wurde das Pulver bei $110^{\circ}$ zur Gewichtsconstanz gebracht. 
Die C- und H-Bestimmung geschah unter Anwendung von Kupferoxyd und Bleichromat und vorgelegter reducirter Kupferspirale.

$0,2590 \mathrm{~g}$ Substanz gaben $\mathrm{CO}_{\mathrm{z}}=0,5106 \mathrm{~g}, \mathrm{H}=0,1673 \mathrm{~g}$, entsprechend $\mathrm{C}=53,77 \%, \mathrm{H}=7,18 \%$.

$0,2156 \mathrm{~g}$ Substanz gaben $\mathrm{CO}_{\mathrm{z}}=0,4233 \mathrm{~g}, \mathrm{H}=0,1452 \mathrm{~g}$, entsprechend $\mathrm{G}=53,55 \%, \mathrm{H}=7,48 \%$.

Die Stickstoff bestimmungen wurden nach Dumas ausgeführt:

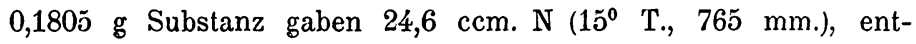
sprechend $16,09 \% \mathrm{~N}$.

$0,2487 \mathrm{~g}$ Substanz gaben $34,5 \mathrm{ccm}$. N (14 $4^{0} \mathrm{~T}$., $\left.751 \mathrm{~mm}.\right)$, entsprechend $16,09 \% \mathrm{~N}$.

Zur Bestimmung des S wurde die Substanz mit rother, rauchender, selbstverständlich auf Schwefelsäurefreiheit geprüfter Salpetersäure oxydirt. Es wurden Bombenrohre von Schott benutzt und auf Temperaturen bis $250^{\circ}$ gegangen. Es schien mir auffällig, wie langsam die starke Salpetersäure angriff. Während Eieralbumin sofort unter Bildung rother dicker Nebel in Lösung ging, blieb das Hämocyanin unter den gleichen Bedingungen fast unverändert. Bei der Verarbeitung des Bombeninhalts erhielt ich auch jedesmal einen gut krystallisirenden S-freien Körper, der sich in Alkali leicht löste. Die Mengen, die ich bisher bekam, waren zu gering, um etwas Genaueres aussagen zu können. Jedenfalls ist der Körper mit keinem der beschriebenen Spaltungsprodukte identisch, worüber die Arbeit v. Fürth: «Einwirkung von Salpetersäure auf Eiweissstoffe ${ }^{1}$ ), Aufschluss gibt. Sobald ich etwas mehr Material gesammelt habe, hoffe ich darüber berichten zu können.

$0,5057 \mathrm{~g}$ Substanz gaben $0,0314 \mathrm{~g} \mathrm{BaSO}_{4}$, entsprechend $0,85 \% \mathrm{~S}$.

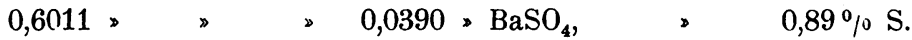
0,7165 * $>0,0446 * \mathrm{BaSO}_{4}, \quad 0,85 \% \mathrm{~S}$.

Die dritte S-Bestimmung wurde mit Hämocyanin gemacht, welches direkt durch sorgsame Dialyse des Blutes erhalten worden war. Die Uebereinstimmung mit den beiden ersten Zahlen dürfte gleichzeitig mit zum Beweis dienen, dass

I) Habilitationsschrift, Strassburg 1899 . 
wir es im Blute von Octopus thatsächlich nur mit Hämocyanin zu thun haben und kein anderer Eiweisskörper darin enthalten ist.

Die vier quantitativen Kupferbestimmungen werden die Anwesenheit des Kupfers im Hämocyanin hoffentlich ausser allen Zweifel stellen. Die beiden ersten Analysen sind durch vorsichtiges Veraschen und Abrauchen des Hämocyanins mit Salpetersäure gemacht worden. Für die beiden letzten Analysen wurde das Kupfer aus den Filtraten der Schwefelsäurefällung durch Schwefelwasserstoff abgeschieden. Das ausgewaschene Sulfid wurde im Porzellantigel geglüht. Diese Methode gilt bei kleinen Mengen als völlig exact. ${ }^{1}$ )

$0,4424 \mathrm{~g}$ Substanz gaben $0,0022 \mathrm{~g} \mathrm{CuO}$, entsprechend $0,39 \% \mathrm{Cu}$. $0,7652 》>0,0031>\mathrm{CuO}, \quad 0,32 \% \mathrm{Cu}$. 0,5057 * $, 0,0024 \cdot \mathrm{CuO}, \quad \rightarrow \quad 0,38 \% \mathrm{Cu}$. 0,6011 > > $0,0028 \rightarrow \mathrm{CuO}, \quad 0,37 \% \mathrm{Cu}$.

Die analytischen Resultate sind nochmals in folgender Tabelle zusammengestellt:

$\begin{array}{rrrlll}\text { C } & 53,77 & 533,55 & & \\ \text { H } & 7,18 & 7,48 & & \\ \text { N } & 16,09 & 16,09 & & \\ \text { S } & 0,85 & 0,89 & 0,85 & \\ \text { Cu } & 0,39 & 0,38 & 0,32(?) & 0,37 \\ \text { O } & 21,72 & 21,61 . & & \end{array}$

Im Mittel enthält also das Hämocyanin: $0=21,67 \%$.

$\mathrm{C}=53,66 \%, \mathrm{H}=7,33 \%, \mathrm{~N}=16,09 \%, \mathrm{~S}=0,86 \%, \mathrm{Cu}=0,38 \%$,

Verhalten des Hämocyanins zu Salzsäure.

In den Lehrbüchern, in denen das Hämocyanin überhaupt erwähnt wird, ist meist angegeben, dass dasselbe durch Salzsäure eine Spaltung völlig im Sinne des Hämoglobins erleide. Es bilde sich ein dem Globin entsprechender Eiweisskörper und zweitens ein krystallisirendes kupferhaltiges Spaltungsprodukt, welches also dem Hämatin an die Seite zu stellen

1) Vergl. Friedheim, Leitfaden für die quantitative chemische Analyse. v. Müller-Kiliani, Kurzes Lehrbuch der analytischen Chemie. 
wäre. Diese Angabe stammt von Fredericq, der sowohl der Salzsäure als auch der Salpetersäure ein gleiches Verhalten zuschreibt. Diese Beobachtung interessirte mich besonders, und ich habe gerade ihr specielle Sorgfalt zugewendet, ohne sie jedoch bestätigen $\mathrm{zu}$ können.

Um sicher zu gehen, habe ich von vornherein verhältnissmässig grosse Mengen Hämocyanin verarbeitet. Die Versuche wurden in der Weise ausgeführt, dass zu der Hämocyaninlösung tropfenweise nicht zu dünne Salzsäure zugefügt wurde. Unter Verschwinden der Blaufärbung fällt ein weisser, flockiger Körper aus. Derselbe liess sich ziemlich schwer filtriren. Das eiweissfreie Filtrat wurde auf dem Wasserbad eingeengt, sobald aber Salzsäuredämpfe entwichen, in den Vacuumexsiccator über Calciumoxyd gebracht, damit nicht so leicht Zersetzungen eintreten sollten. In dieser Weise wurde zweimal mit krystallisirtem und dialysirtem Hämocyanin gearbeitet. Das erste Mal hinterblieb eine ganz geringe Menge weisser Nädelchen, die in einer grünlichgelben Mutterlauge eingebettet waren. Ich glaubte schon, dass die Spaltung trotz der verschwindenden Menge im Sinne der Hämoglobinspaltung verlaufen wäre. Es war jedoch nicht der Fall. Die Nädelchen waren sehr schwer in Wasser löslich und gaben die Reaction auf Schwefelsäure und auf Calcium. Es war demnach Gyps, der vielleicht von dem destillirten Wasser bei der Dialyse herrührte. Kupfer liess sich sehr scharf in der erwähnten Mutterlauge nachweisen (Ferrocyankalium, Ammoniak, Schwefelwasserstoffreactionen). Ein zweiter Versuch mit krystallisirtem Hämocyanin verlief ebenso resultatlos, desgl. eine Probe mit direkt durch Dialyse aus Blut gewonnenem Hämocyanin. Durch diese Versuche war zunächst erwiesen, dass das Hämocyanin zwar eine Zersetzung erleidet, bei der das Kupfer abgespalten wird. Der gleichzeitig entstehende Eiweisskörper ist frei von diesem Metall. Er zeigt alle Eigenschaften eines Acidalbumins. Er war schwer löslich in Wasser bei Gegenwart von Salzsäure. Bei anhaltender Dialyse wurde er gallertartig gefällt. Die Lösungen waren leicht fällbar durch alle Neutralsalze etc. Etwa einen kupferfreien nativen Eiweisskörper darzustellen, 
gelang mir nicht. Ich versuchte unter anderem die Methode von Schulz ${ }^{1}$ ) zur Gewinnung des Globins aus Hämoglobin anzuwenden.

Ich habe auch eine Analyse des Acidalbumins ausgeführt, um zu sehen, ob sich etwa in der elementaren Zusammensetzung eine grosse Abweichung zeigen würde. Auch dies war nicht der Fall.

Der salzsäurefrei dialysirte Eiweisskörper wurde gewaschen und schliesslich bei $110^{\circ}$ getrocknet.

$$
\begin{gathered}
0,1611^{\circ} \mathrm{g} \text { Substanz gaben } 22,5 \mathrm{ccm} . \mathrm{N}\left(18,0^{\circ} \mathrm{t} ., 757 \mathrm{~mm} .\right), \\
\text { entsprechend } 16,04 \% \mathrm{~N} . \\
0,1953 \mathrm{~g} \text { Substanz gaben } 0,3796 \mathrm{~g} \mathrm{CO}_{2}, 0,1343 \mathrm{H}_{2} \mathrm{O}, \\
\text { entsprechend } 53,01 \% \mathrm{C}, 7,64 \% \mathrm{H} .
\end{gathered}
$$

Es erübrigt noch, ein Wort über die Bindung des Kupfers im Hämocyanin zu sagen. Wenn man den trockenen pulverisirten Eiweisskörper mit Ferrocyankalium befeuchtet, so ist absolut keine braunrothe Färbung $\mathrm{zu}$ bemerken (Bildung von Ferrocyankupfer). Es genügt jedoch, denselben vorher mit etwas 1\% starker Salzsäure zu befeuchten, um in wenigen Augenblicken die genannte Färbung auftreten zu sehen, die immer mehr an Intensität zunimmt. Genau so wirkt eine 1\%ige Essigsäurelösung.

Reine Hämocyaninlösungen verh lten sich genau so. Direkt liefern sie keine Kupferaction. Setzt man vorsichtig ganz verdünnte Salzsäure zu, so entsteht, wie früher angeführt, ein Niederschlag. In diesem Momente zeigt Ferrocyankalium noch kein Kupfer an. Erst in dem Momente, wo weiterer Salzsäurezusatz den Niederschlag gerade wieder gelöst hat, tritt die Reaction ein.

Aus dieser Thatsache folgt, dass das Kupfer nicht in fester organischer Bindung, etwa wie das Eisen, im Hämoglobin vorhanden ist, sondern sehr lose an clen Eiweisskörper gebunden ist. Wir haben also im Hämocyanin eine Verbindung nach Art eines Kupferalbuminates vor uns.

1) Diese Zeitschr., Bd. XXIV, S. 449. 
Das Sauerstoffbindungsvermögen des Hämocyanins.

Auf die wichtige respiratorische Fähigkeit des Hämocyanins hat wohl Bert ${ }^{1}$ ) zuerst aufmerksam gemacht. Fredericq beschreibt den Vorgang genauer bei Octopus in seiner citirten Arbeit. Er unterscheidet auch in Analogie zum Hämoglobin und Oxyhämogkobin ein Hämocyanin und Oxyhämocyanin. Wie schon Eingangs erwähnt, lässt sich die mit der Sauerstoffaufnahme verbundene Farbenänderung nicht nur ausgezeichnet am lebenden Thier, sondern auch an dem der Arterie entnommenen Blute beobachten. Letzteres wird beim Durchleiten indifferenter Gase, wie Wasserstoff, Kohlensäure, Stickstoff etc. entfärbt (vergl. Krukenberg). Das gleiche Phänomen erzeugen die üblichen Reductionsmittel, Schwefelammon, Natriumhydrosulfit etc. Beim Stehen des Blutes an der Luft verschwindet die blaue Farbe gleichfalls. Nur die oberste, direkt mit der Luft in Berührung kommende Schicht behält dieselbe bei.

Obwohl nun Heim diesen Farbenwechsel des Blutes zugibt, bestreitet er jedoch, dass das Hämocyanin den Sauerstoffüberträger vorstelle. Er habe nie mehr Sauerstoff beim Auspumpen des Blutes im Vacuum erhalten, als dem Sauerstoffgehalte des reinen Wassers entspräche. Es finden sich jedoch keine Zahlenbelege dafür.

Die gleiche Behauptung machen Jolyet und Regnard. Heim citirt in seiner Abhandlung deren Worte «la teneur en oxygène du sang des animaux aquatiques est égale à la teneur en oxygène de l'eau ambiante.» Mir war die Originalarbeit der beiden französischen Forscher leider nicht zugänglich. Nun ist, wie man leicht berechnen kann, reines Wasser im Stande, ca. $0,6 \%$ Sauerstoff bei ca. $15^{\circ}$ C. aus der Luft zu absorbiren, Blut natürlich noch viel weniger, vergl. Hüfner (Du Bois, Arch. 1895, 102). Jolyel und Regnard selbst finden, wie unten genauer angegeben, durchschnittlich $3 \frac{1}{2} \%$ Sauerstoff. Es widersprechen also die Thatsachen selbst ihrer Meinung.

1) «Mémoire sur la physiologie de la seiche» in Mémoires de la Société scientif. de Bordeaux. 1867.

Auch in: Leçons sur la physiologie comparée de la respiration, pag. 149. 1870. 
Wasser vermag wohl aus einer reinen Sauerstoffatmosphäre 3,5\% Sauerstoff zu absorbiren, aber selbstverständlich haben wir hier nur mit dem Partialdruck des Sauerstoffes der Luft zu rechnen.

In der Litteratur ist mir nur eine Angabe über den quantitativen Gasgehalt des Octopusblutes aufgestossen. Dieselbe stammt von Griffiths, ${ }^{1}$ ) der auch andere in ihrem Blute Hämocyanin führende Thiere daraufhin uritersuchte. Der Procentgehalt an Sauerstoff der verschiedenen Blutarten (Octopus, Sepia, Pagurus, Palinurus, Homarus, Acherontia) schwankt zwischen 13-16. Dieser hohe Sauerstoffgehalt ist sehr auffällig, trotzdem die Parallelanalysen ganz ausgezeichnet übereinstimmen. Nicht nur auf die angegebene Aeusserung Heim's hin dürfte man an ihrer Richtigkeit zweifeln, sondern auch die eben genannte Untersuchung von Jolyet und Regnard aus dem Jahre 1877 bestärkt darin. Während z. B. Griffiths für Pagurus 14,79, 14,88, 14,96, 14,85\% erhält, finden Jolyet und Regnard 2,4 und 4,4\%.

Ich war leider aus Mangel an Zeit nicht mehr im Stande, die Gasanalyse an reinem krystallisirten Hämocyanin zu machen. Da aber im Octopusblut kein anderer Körper der Sauerstoffüberträger sein kann, ausser dem allein darin enthaltenen Hämocyanin, so erlaubt der aus dem Blute auspumpbare Sauerstoff direkt einen Schluss auf das Sauerstoffbindungsvermögen des Hämocyanins. Die Bestimmungen wurden mit der Luftpumpe unter gleichzeitiger Erwärmung des Blutes auf $50^{\circ}$ gemacht. Rücksicht auf gebundene oder freie Kohlensäure wurde nicht genommen. Die Kohlensäure wurde durch Aetzkalium, der Sauerstoff durch Pyrogallol absorbirt.

I.

Angewandte Blutmenge $39 \mathrm{ccm}$ :

Gefundenes Gas $13,4 \mathrm{ccm} .\left(25^{\circ} \mathrm{t}, 753 \mathrm{~mm}\right.$. B., minus $\left.273 \mathrm{~mm} . \mathrm{Hg}-\mathrm{Säule}\right)$ Nach $\mathrm{CO}_{2}$-Absorption 6,0 > > 321 , ,

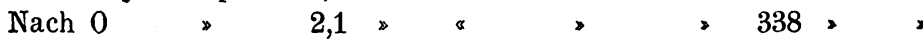

1) Proc. R. S. Edinburgh 18. 288. 
Nach Reduction auf $0^{\circ}$ und $760 \mathrm{~mm}$. Barom.-St. sind demnach vorhanden:

$$
\begin{aligned}
\mathrm{CO}_{2} & =3,41 \mathrm{ccm} . \\
0 & =1,44 \\
\mathrm{~N} & =0,75 \\
\text { Summa } & =5,60 \mathrm{ccm} .
\end{aligned}
$$

Oder $100 \mathrm{ccm}$. Blut enthalten:

$$
\begin{gathered}
\mathrm{CO}_{2}=8,74 \% \\
\mathrm{O}=3,70 \\
\mathrm{~N}=1,92
\end{gathered}
$$

II.

Angewandte Blutmenge $36 \mathrm{ccm}$. :

Gefundenes Gas $\quad 12,8 \mathrm{ccm}$. ( $26^{\circ} \mathrm{t}, 759 \mathrm{~mm}$. B., minus $279 \mathrm{~mm}$. Hg-Säule) Nach $\mathrm{CO}_{2}$-Absorption 6,4 > 》 323 " "

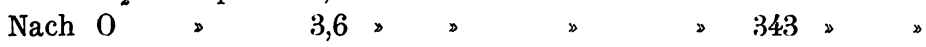

Bei Reduction auf $0^{\circ}$ und $760 \mathrm{~mm}$. Barom.-St. sind demnach vorhanden:

$$
\begin{aligned}
\mathrm{CO}_{2} & =2,92 \mathrm{ccm} . \\
0 & =1,11 \\
\mathrm{~N} & =1,29 \\
\text { Summa } & =5,32 \mathrm{ccm} .
\end{aligned}
$$

Oder $100 \mathrm{ccm}$. Blut enthalten:

$$
\begin{gathered}
\mathrm{CO}_{2}=8,11 \% \\
\mathrm{O}=3,09 \\
\mathrm{~N}=3,58
\end{gathered}
$$

Ein kurzer Ueberblick zeigt also, dass $100 \mathrm{ccm}$. Blut durchschnittlich $3,4 \mathrm{ccm}$. Sauerstoff enthalten. Andererseits beträgt der Hämocyaningehalt des Octopusblutes $9 \%$. Es würde demnach $1 \mathrm{~g}$ Hämocyanin ca. 0,4 ccm. Sauerstoff zu binden vermögen, was ungefähr dem vierten Theile des Oxyhämoglobins entspräche.

Neapel, Juli 1901. 